\title{
Design of the Hydrostatic Thrust Spherical Bearing with Restrictors (Fitted Type)
}

\author{
Ahmad Waguih Yacout Elescandarany \\ Mechanical Department, Faculty of Engineering, Alexandria University, Alex, Egypt \\ Email address: \\ awaguih $@$ yahoo.com

\section{To cite this article:} \\ Ahmad WaguihYacout Elescandarany. Design of the Hydrostatic Thrust Spherical Bearing with Restrictors (Fitted Type). International \\ Journal of Mechanical Engineering and Applications, Vol. 7, No. 2, 2019, pp. 34-45. doi: 10.11648/j.ijmea.20190702.11
}

Received: March 1, 2019; Accepted: April 26, 2019; Published: May 29, 2019

\begin{abstract}
The hydrostatic bearings are broadly observed in industrial applications owing to their potential characteristics of low running friction, high load-carrying capacity, high stiffness and small viscous dissipation. Spherical bearings have advantages over other configurations of bearings because of their self-aligning property and also their capacity to accept both radial and thrust loads. Operation of the spherical bearings is unaffected by angular miss-alignment. They are mainly used in machine tools, precision measuring instruments, hydraulic piston pumps and motors, gyroscope gimbals, telescopes, radar tracking units, air craft engines and rotary drum of centrifugal separator for enriched uranium. The main objective of this part of study is to handle the fitted bearing design taking into consideration the combined effects of rotational inertia, surface roughness and the fluid film viscosity variability. The mathematical expressions derived in part 2 for this type of bearings with its different configurations (with and without recess; hemispherical and partial hemispherical seats) have been used in the detailed bearing design. Improving the previous design through developing some mathematical expressions, a new conception is adopted to reveal the bearing consistency showing how the fluid flow rate pulsation or intermittent could be prevented. The detailed design procedures show the advantages of this method to get the central pressure ratio mathematically through maximizing the flow rate with minimizing the supply pressure which means minimum pump power to get maximum discharge. This design showed very high mean static stiffness of all the bearing configurations and an acceptable temperature rise for a heavy duty bearing without the need to a use a cooling system.
\end{abstract}

Keywords: Hydrostatic Bearings, Spherical Bearings Design, Surface Roughness, Inertia, Viscosity Effects

\section{Introduction}

Nowadays humanity is much more dependent upon the mechanical machines for their daily needs. To take care of smooth operations of the relatively moving surfaces of the machine without wear and tear, many different bearing types and configurations (dynamic and hydrostatic) have been designed. Hydrostatic bearing design requires adjustment of a number of parameters including the bearing geometry, restrictor size and type, supply pressure, central pressure, surface roughness, rotational speed, lubricating fluid properties and bearing eccentricity to optimize performance. The flow restrictor is necessary to provide a pressure drop between the supply and the inlet pressures, where design of flow restrictors influences bearing stiffness, pumping power, supply pressure, and lubricant flow. While the load carrying capacity is dependent on the bearing geometry, eccentricity, rotational speed, surface roughness, fluid viscosity and the central pressure it is very difficult to design a robust, consistent and highly stiffened thrust spherical bearing.

Dowson and Taylor [1] studied the hydrostatic spherical bearing with adiabatic lubricant flow considering the inertia effect on the bearing performance concluding that the inertia affects positively the fitted bearing performance and the adiabatic temperature rise decreases with the increase in eccentricity pointing to neither the central pressure ratio nor the bearing static stiffness.

Row [2-3] studied and designed the externally pressurized bearing concluding that the multi-recess bearing is usually preferred than that of the single recess and the design pressure ratio should be 0.5 for the spherical bearing considering lubricant constant viscosity without pointing to the bearing stiffness.

Shijiang and Decheng [4] studied the spherical bearing 
with what is called a pressure tracing system which is suitable for heavy duty forging presses; it is concluded that in practical designs throttling ratio (pressure ratio inverse) should be greater than 1.5 , i.e. the inlet pressure should be less than $(2 / 3)$ the supply pressure and this pressure tracing system results in more stiffened bearing.

Toshiharu Kazama [5] offered an optimal bearing design concluded that for the fitted type of thrust spherical bearings the optimum design pressure ratios are (2/3 and 0.6907) for capillary tube and orifice restrictors respectively without pointing to the bearing stiffness.

Satish C. Sharma et al [6 -7] described theoretically the performance of circular and journal hydrostatic bearings having recesses of different geometric shapes. The computed results indicated that: to get an improved performance a proper selection of the geometric shape of the recess in conjunction with the type of restrictor and the value of the restrictor design parameter is essential.

Lin and Chiang [8] studied and designed the hydrostatic circular thrust bearing showing that the central pressure ratio could be between 0.5 and 0.7 and the stiffness depends on the restrictor parameter, radius ratio and the film height.

Ahmad W. Yacout et al [9] studied the fitted hydrostatic thrust spherical bearing considering the combined effects of the inertia and the surface roughness on the bearing performance showing that the central pressure ratio is $(2 / 3)$ regardless the restrictor type and offering a bearing design based on minimum losses and optimal restrictor dimensions.

$\mathrm{Lu}$ Lihua et al [10] proposed a fluid-structure interaction model to calculate the static performance of hydrostatic bearing where the temperature rise, the pressure distribution and static stiffness were calculated by computational fluid dynamics (CFD). When comparing the calculated results with those got empirically, the static stiffness showed $78 \%$ drop.

Elescandarany [11-12] studied the effect of the fluid viscosity variation on the fitted hydrostatic thrust spherical bearing performance with and without recess taking into consideration the effects of the inertia and the surface roughness showing that the central pressure ratio is $(2 / 3)$ and offering a bearing design with temperature rise less than 14 degrees centigrade, in addition to a comparison between the two bearings designed in Yacout et al [9] and in Elescandarany [11-12].

Due to the lack of theoretical researches handling the detailed design of such type of bearings taking into consideration the effects of the surface roughness, the inertia, lubricant variable viscosity and the bearing geometry which could not only be difficult but also tedious, the present study offers detailed procedures and calculations to design a robust, consistent and highly stiffened fitted hydrostatic thrust spherical bearing with its different configurations using a capillary tube and orifice restrictors revealing the mathematical relations between the seat dimensions and the eccentricity and determining the maximum central pressure ratio which gives the bearing its consistency, the localized static stiffness and its mean value, the geometry factor and the temperature rise and distribution ' theoretically and numerically' where all these items were absent in the author previous studies [9, 11-12]. All bearing parameters were calculated for the different bearing configurations using both restrictors to enable the selector choosing the most appropriate configuration serving his purposes.

\section{Design Considerations}

There are five groups of factors should be considered when this type of bearings is designed:

Geometrical group $\left(\varphi_{i}, \varphi_{r}, \varphi_{e}, \eta\right)$

Manufacturing group $(\sigma, \xi, \Lambda)$

Operational group $\left(N, w, e, \beta, p_{i,} p_{s}, q, t, \lambda\right)$

Fluid group $\left(\mu, K_{v}, \rho, C_{v}\right)$

Restrictors dimensions $\left(d_{c}, l_{c}\right)$ or $\left(d_{o}, C_{d}\right)$

\subsection{Bearing Design Data}

\subsubsection{Submitted Data}

The bearing designer could be provided with: (L, $N$ and $R$ )

\subsubsection{Selected Data}

Selecting the geometrical group to be:

$\left(\varphi_{i}, \varphi_{r}, \eta \geq 201, m_{c}, m_{o}\right)$

Selecting the manufacturing group:

$(\Lambda=5 \rightarrow 100$ or $\xi$, $=0.05 \rightarrow 1)$

Selecting the fluid group to be for instance:

$\mu=0.086 \mathrm{~N} . \mathrm{s} / \mathrm{m}^{2}, K_{v}=0.5$,

$\rho=867 \mathrm{~N} . \mathrm{s}^{2} / \mathrm{m}^{4}, \mathrm{C}_{\mathrm{v}}=1880 \mathrm{~J} / \mathrm{Kg} . \mathrm{C}^{\mathrm{o}}$

\subsubsection{Calculated Data}

Operational group could be calculated.

$\left(S, W, e, \beta, P_{s}, P_{i}, d_{c}, l_{c}, d_{o}, t, \lambda\right)$

\subsection{Design Mathematical Expressions}

The mathematical expressions governing the bearing design and performance could be found in the Appendix.

\section{Design Procedures}

It is necessary to have a comprehensive idea about the sequence of the design steps depending on the data provided, data selected and data calculated.

\subsection{Central Pressure Ratio Determination ( $\beta)$}

One of the design difficulties is determining the central pressure ratio mathematically, putting aside the industrial experience which allows the designer to select its value to be between (0.5and 0.7 ), it will be derived mathematically when using a capillary and orifice restrictors showing the drawbacks of selecting it by experience.

\subsubsection{Capillary Tube Restrictor}

Following Yacout [9], the derivation could be found in the Appendix.

\subsubsection{Orifice Restrictor}

Following Yacout [9], the derivation could be found in the 
Appendix.

\subsection{Speed Parameter Determination (S)}

Once the supply pressure $\left(p_{s}\right)$ is determined, the speed parameter $(S)$ could be calculated as from Yacout [9] as:

$S=3 \rho \Omega^{2} R^{2} / 40 p_{i}$

Where:

$\Omega=2 \pi N \& p_{i}=\beta p_{s}$

In turn, the dimensionless load carrying capacity $(W)$, flow rate $(Q)$ and friction torque $(M)$ could also be calculated at any given eccentricity $(e)$

\subsection{Eccentricity Determination (e)}

Calculating $(M)$ and $(Q)$ at any (e) allows calculating the pump power $\left(P_{p}\right)$, frictional power and the total power loss $\left(P_{t}\right)$ at different values of $(e)$ as from [9] and from the appendix equations (8-10):

$$
\begin{aligned}
& P_{p}=\left(\frac{\pi \beta p_{s}^{2} e^{3}}{6 \mu}\right) Q \\
& P_{f}=\left(\frac{2 \pi \mu R^{4} \Omega^{2}}{e}\right) M \\
& P_{t}=P_{f}+P_{p}
\end{aligned}
$$

Then eccentricity (e) that gives the minimum total power losses could be graphically determined.

\subsection{The Pressure Distribution (P)}

Once determining the eccentricity (e) and the speed parameter $(S)$, the pressure distribution could be calculated by equitation (2).

\subsection{Load Carrying Capacity Determination (w)}

Getting the eccentricity, speed parameter and the central pressure ratio $(\beta)$ the dimensionless load carrying capacity $(W)$ could be calculated using equation (5), in turn the load (w) as:

$$
\mathrm{w}=\pi \mathrm{R}^{2} \beta \mathrm{P}_{\mathrm{s}} \mathrm{W}
$$

\subsection{Temperature Rise}

Temperature rise could be determined theoretically as:

$$
\Delta T=\frac{P_{t}}{\rho m_{f} C_{v}} \text { or numerically as in equation (7). }
$$

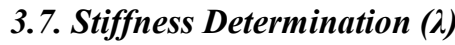

The bearing stiffness could be numerically calculated using equations (11-14).

\subsection{The Bearing Consistency}

From Equations $(18,22)$ :

The quantities $a_{1}=\frac{72 n_{c} \mu^{6} \mathrm{Re}_{c}^{3}}{e^{6} \rho^{3} p_{s}^{3} Q^{2}} \& a_{2}=\frac{9 C_{d}^{2} \mu^{6} \mathrm{Re}_{o}^{4}}{8 e^{6} \rho^{3} p_{s}^{3} Q^{2}}$, for the capillary and orifice restrictors respectively should be
(4/27) to avoid the existence of two central pressure values which makes the bearing inconsistent i.e. the central pressure ratio should be (2/3)to get a consistent bearing Figure 1 .

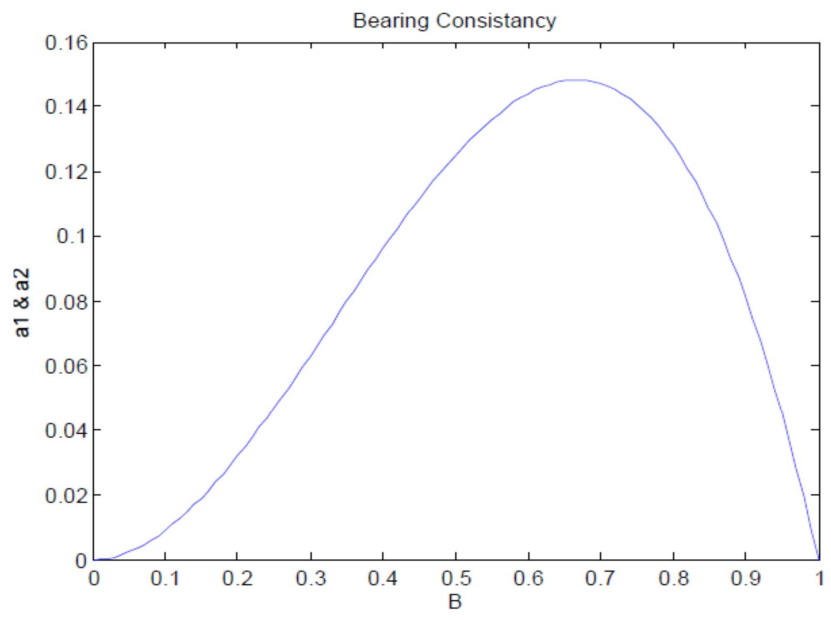

Figure 1. Bearing Consistency.

\section{Design Example}

It is required to design a bearing with a radius of $(50 \mathrm{~mm})$, maximum rotor speed $(N=300 r . p$. s) and lifting a load of $(L$ $=40 \mathrm{KN}$ ).

\section{Design Example Calculations}

For this example; a bearing with its four different configurations has been designed to find out which one is the best for this case. Table 1 .

It is preferable to choose one bearing configuration to reveal and perform the design procedures and calculations.

A recessed hemispherical configuration is suggested.

\subsection{Bearing Configuration and Lubricant Fluid Data}

Geometrical group:

$\left(\varphi_{i}=5^{o}, \varphi_{r}=24^{o}, \varphi_{e}=(\pi \eta / 2), \eta=1\right)$

Manufacturing group: $(\xi=0.05)$

Restrictor data as put in the theoretical analysis $\left(C_{d}=0.6\right.$ for orifice, $n_{c}=20$ for capillary and $R e=2000$ )

Fluid group:

$\left(\mu=0.086 \mathrm{~N} . \mathrm{s} / \mathrm{m}^{2}, K_{v}=0.5, \rho=867 \mathrm{~N} . \mathrm{s}^{2} / \mathrm{m}^{4}, C_{v}=1880\right.$ $\left.\mathrm{J} / \mathrm{Kg} \cdot \mathrm{c}^{o}\right)$

\subsection{The Bearing Geometry Factor Determination $\left(J_{f}\right)$}

The dimensionless load carrying capacity $(W)$ in the stationary state is less than the unity depending on the bearing configuration. Hence the geometry factor is $(1 / W)$.

\subsection{Determination of the Primary Supply Pressure and Speed Factor $\left(p_{l} \& S_{l}\right)$}

$$
\begin{aligned}
& p_{l}=\frac{L}{\beta \pi R^{2}}=\frac{3 \times\left(40 \times 10^{3}\right) \times\left(10^{4}\right)}{2 \times 25 \times \pi} \\
& p_{l}=7.6394(10)^{6} \mathrm{~N} / \mathrm{m}^{2}
\end{aligned}
$$




$$
\begin{aligned}
& S_{l}=\frac{3 \rho \Omega^{2} R^{2}}{40 \beta p_{l}}=\frac{3 \times 867 \times(2 \pi \times 300)^{2} \times(0.05)^{2}}{40 \times(2 / 3) \times 7.6394(10)^{6}} \\
& S_{l}=0.1134
\end{aligned}
$$

\subsection{Determination of the Primary Eccentricity and Dimensional Load Capacity $\left(e_{l} \& W_{l}\right)$}

Applying equations $(3,6)$ using the primary speed factor determined $(0.1134)$, the parameters $(Q, M)$ could be calculated at different values of $(e)$ in turn $(P f, P p, P t)$ could be also be calculated and drawn using equations (8-10); And graphically the $\left(e_{l}\right)$ corresponding to the minimum power losses $(P t)$ could be determined to be:

$$
\mathrm{e}_{\mathrm{l}}=1.5466 \mathrm{~mm}
$$

Getting this primary eccentricity the exit flow angle $\left(\theta_{e l}\right)$ could be determined from the appendix (A1.1) to be: $\left(\theta_{e l}=\right.$ $88.23^{\circ}$ ), in turn the dimensionless load capacity $\left(W_{\nu}\right)$ could be calculated at the stationary state $(N=0)$ using equation (5) to be: $\left(W_{l}=0.9924\right)$
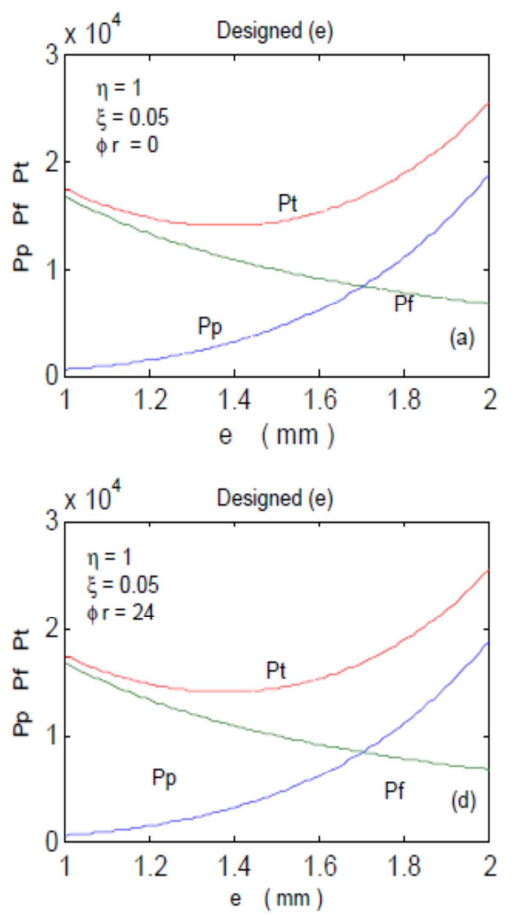

Figure 2. Eccentricity determination.

\subsection{Load Carrying Capacity (W)}

As before $\left(\theta_{e}\right)$ and $(S)$ could be calculated to be:

$\theta_{e}=88.42^{\circ}$ and $\mathrm{S}=0.1126$; in turn the dimensionless load capacity $(W)$ could be calculated at $(\mathrm{S}=0 \& 0.1126$ ) to give
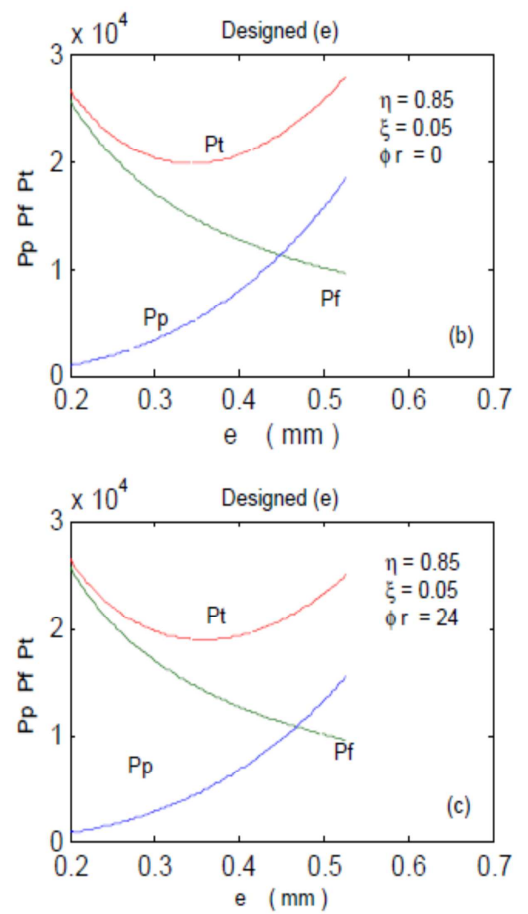

Hence, the bearing geometry factor $\left(J_{f}\right)$ is:

$$
J_{f}=1 / W_{l}=1 / 0.9924=1.0076
$$

\subsection{The Design Supply Pressure Evaluation (ps)}

The design supply pressure which could lift the load $(L)$ at the stationary state $(N=0)$ is:

$$
p_{s}=J_{f} \times p_{l}=1.0076 \times 7.6394(10)^{6}
$$

Then:

$$
p_{s}=7.69785(10)^{6} \mathrm{~N} / \mathrm{m}
$$

\subsection{The Design Eccentricity Determination (e)}

Determining the design supply pressure, the design eccentricity could be determined graphically as before from figure (2-c) to be: $(e=1.4 \mathrm{~mm})$.

( $W_{s=0}=0.9924$ and $W_{s=0.1126}=1.1017$ ) at the supply pressure $\left(p_{s}\right)$ respectively.

Hence:

$$
\begin{aligned}
& w_{s=0}=\pi R^{2} \beta p_{s} W_{s=0}=L=\pi x(0.05)^{2} x(2 / 3) \times 7.6975(10)^{6} x(0.99241)=40 K N \\
& w_{s=0.1126}=\pi R^{2} \beta p_{s} W_{s=0.1126}=\pi x(0.05)^{2} \times(2 / 3) \times 7.6975(10)^{6} \times 1.1017 \\
& w_{s=0.1126}=44.4 K N
\end{aligned}
$$


Hence, this ultimate load should not be exceeded.

\subsection{The Lubricant Fluid Flow Rate (q)}

From equations (3-4) the dimensionless flow rate $(\mathrm{Q}=0.003)$ and:

$$
\begin{aligned}
& q=\pi e^{3} \beta p_{s} Q / 6 \mu=\frac{(3.14)(0.00155)^{3}(2 / 3)\left(5.1408 \times 10^{6}\right)(0.0051)(3600)}{6(0.068)} \\
& q=1.7994 \mathrm{~m}^{3} / \mathrm{hr}
\end{aligned}
$$

\subsection{Restrictor Dimensions Calculation}

(i) Capillary tube

From equation (24):

$$
\begin{aligned}
& K_{c}=\frac{3 q \mu}{p_{s}}=\frac{128 d^{3}}{n_{c}} \\
& d=\left(\frac{3 n_{c} q \mu}{128 p_{s}}\right)^{\frac{1}{3}} \\
& =\left[\frac{3 \times 20 \times\left(1.12 \times 10^{9}\right) \times(0.068)}{3600 \times 128 \times\left(7.697 \times 10^{6}\right)}\right]^{\frac{1}{3}} \\
& d_{c}=1.9 \mathrm{~mm} \\
& l=1.9 \times 20=38 \mathrm{~mm}
\end{aligned}
$$

(ii) Orifice

From equation (25):
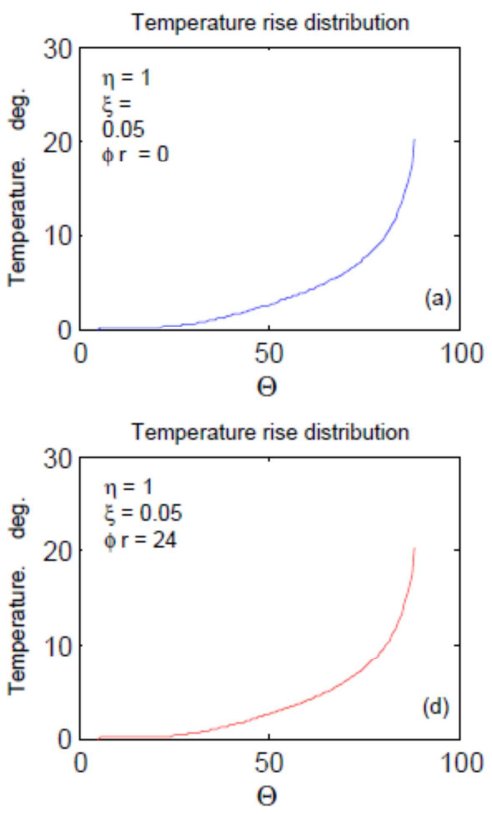

$$
\begin{aligned}
& q=K_{o}\left(p_{s} / 3\right)^{1 / 2} \\
& K_{o}=\pi C_{d} d_{o}^{2} / \sqrt{8 x \rho} \\
& d_{o}=\left[q \sqrt{8 x \rho} /\left(\pi C_{d} \sqrt{p_{s} / 3}\right)\right]^{1 / 2} \\
& {\left[\frac{(1.12 / 3600) \times \sqrt{8 \times 867}}{\pi \times 0.6 \times \sqrt{7.697 \times 10^{6} / 3}}\right]^{1 / 2}} \\
& d_{o}=0.0029 \mathrm{~m} \\
& \quad=2.9 \mathrm{~mm}
\end{aligned}
$$

\subsection{Temperature Rise and Stiffness Determination}

The temperature rise could be numerically and theoretically calculated whereas the theoretical stiffness calculation is quite difficult.

\subsubsection{Temperature Rise}

The temperature rise could be numerically calculated through applying equation (7) at the rotational state to be (20.26. C) and theoretically as:

$$
\Delta t=\frac{P_{f /}}{\rho q C_{v}}=\frac{11066 \times 3600}{867 \times 1.457 \times 1880}=21.6 C^{o}
$$

Figure (3-c) shows the temperature rise distribution.
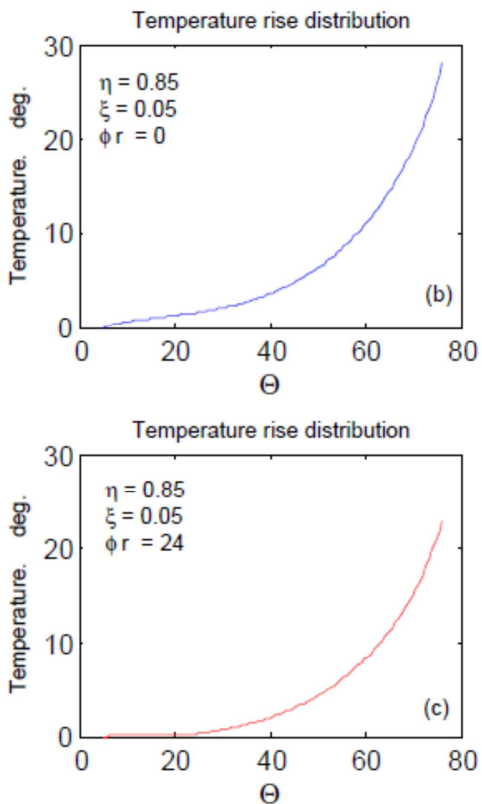

Figure 3. Temperature rise distribution.

\subsubsection{Stiffness Determination ( $\lambda$ )}

The stiffness is calculated numerically through an infinitesimal change in the eccentricity to get the bearing main stiffness at the rotational state as $(203,404 \mu / \mathrm{m})$ with capillary and orifice restrictors respectively, figure (4-c). 

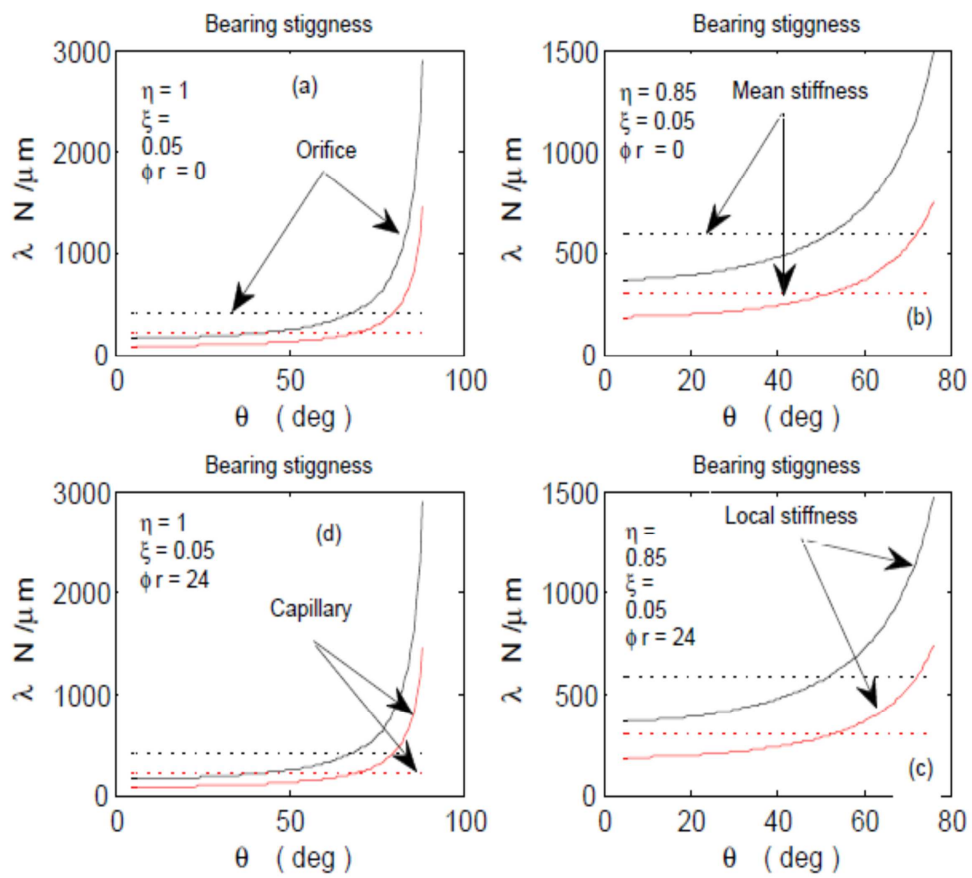

Figure 4. Local stiffness and its mean value.

\section{Results}

This design technique produces a consistent and highly stiffened bearing in addition to a good temperature rise that needn't to any cooling system where the central pressure ratio is $(2 / 3)$,mean stiffness is in range of $(100-400 \mathrm{~N} / \mathrm{m})$ and a temperature rise $(20-28 \mathrm{C})$ for all the bearing configurations.

\section{Discussion}

The bearing is designed based on minimum power losses, minimum flow rate and optimal restrictor dimensions taking into consideration the rotational inertia, the surface roughness and the lubricant viscosity variation $[9,11 \& 12]$.

\subsection{Central Pressure Ratio}

This central pressure ratio has been derived based on previous work of Yacout [9] avoiding the solution of the $3^{\text {rd }}$ order equations $(18,22)$ where their solution haven't given a conception about the bearing consistency. The present study offered a new idea about these equations, through their differentiation, where equating their $1^{\text {st }}$ derivative, relating to the central pressure ratio, and equating to zero have given maximum values of the quantities $(a 1, a 2)$ to be (4/27) and a unique value(2/3) for the central pressure ratio. Rowe and stout [2] show that the pressure ratio is (0.4-0.6), Shijiang [4] concluded that it should be $(\leq 2 / 3)$, Kasama [5] showed this ratio as (0.66) and Lin [8] showed that the critical pressure ratio is $(0.4902)$, and the operational value $(0.7)$.

\subsection{Bearing Consistency}

Figure (1) shows the quantities $(a 1, a 2)$ versus the central pressure ratio $(\beta)$ through drawing equations $(18,22)$. It is clear that if the quantities $(a 1, a 2)$ have any values less than (4/27) will result in two values of $(\beta)$ which will make the flow rate severely or slightly changes depending on the difference between these two values causing a pulsating or intermittent fluid flow and could lead to the bearing deterioration due to the change of the load carrying capacity. This can't support Lin [8] in the critical pressure ratio where at $(\beta=0.4902)$ the quantity (a1 or a2)will be about $(0.1224)$ which gives another $(\beta)$ value of about (0.815); the difference between these two ratios is quite big.

\subsection{Temperature Rise}

The temperature rise calculated numerically or theoretically showed approximately the same results which give the priority to the theoretical solution for its simplicity, but the numerical one allows the designer to get an idea about the distribution of this temperature rise figure 3 . Elescandarany [11-12] showed that the temperature rise is about 14 degrees centigrade for a light load capacity (about one tenth of that of the present bearing) and didn't pay attention to the theoretical calculation.

\subsection{Bearing Stiffness}

One of these design technique difficulties is calculating the bearing stiffness where it is a function of the film thickness and the thickness is variable. Hence, the present study offered a new conception about calculating the stiffness numerically where the stiffness is calculated as the mean stiffness of a sample of localized stiffness calculated at a (100) different thicknesses along the bearing (4)shows the local stiffness and its mean one. Rowe [2] designed a hydrostatic spherical bearing with $(53,20 \mathrm{~N} / \mathrm{m})$ average radial and axial stiffness 
respectively while the present design technique produces a bearing of a very high mean stiffness $(200-300 \mathrm{~N} / \mathrm{m})$ and $(400-600 \mathrm{~N} / \mathrm{m})$ for capillary and orifice restrictors respectively according to the bearing configuration.

\subsection{Other Bearing Parameters}

The other different calculations of the chosen bearing configuration could be found in Table 1 .

\subsection{Configuration Selection}

Table 1 shows the details of each configuration to enable the designer selecting the best suitable one for the client required purpose. For instance, a partial hemi-spherical with or without recess, orifice restrictor, has the highest mean stiffness, acceptable flow rate and temperature rise but with more power losses. If the least power losses is needed a hemi-spherical with or without recess, orifice restrictor, could be selected and so on.

\section{Conclusion}

From the previous results and discussion it could be said that the present design technique is reliable and dependable to design a highly consistent and stiffened bearing recommending and preferring the orifice restrictor.

The four bearing configurations have approximately the same quality.

The central pressure ratio should be $(2 / 3)$.

\section{Future Work}

Part 4 will be the last part of this series and will offer a restrictor-less bearing design with a comprehensive analysis of the bearing stiffness.

Table 1. Bearing calculations for the different configurations.

\begin{tabular}{|c|c|c|c|c|}
\hline \multirow{2}{*}{$\begin{array}{l}\text { Bearing } \\
\text { Parameters }\end{array}$} & \multicolumn{2}{|c|}{ Without recess } & \multicolumn{2}{|c|}{ With recess } \\
\hline & H. S & P.H. S & H. S & P. H. S \\
\hline$\xi$ & 0.05 & 0.05 & 0.05 & 0.05 \\
\hline$\eta$ & 1 & 0.85 & 1 & 0.85 \\
\hline$\varphi \mathrm{I}($ degree $)$ & 5 & 5 & 5 & 5 \\
\hline$\varphi r \approx$ & 0 & 0 & 24 & 24 \\
\hline$\varphi \mathrm{e} \approx$ & 90 & 76.5 & 90 & 76.5 \\
\hline$\Delta(\mathrm{mm})$ & 0 & 0 & 5 & 5 \\
\hline $\operatorname{dio} \approx$ & 8.7 & 8.7 & 8.7 & 8.7 \\
\hline $\mathrm{R} \approx$ & 50 & 50 & 50 & 50 \\
\hline $\mathrm{a}(\mathrm{mm}) 2$ & 79 & 79 & 79 & 79 \\
\hline Load (KN) & 40 & 40 & 40 & 40 \\
\hline N(r. p. s) & 300 & 300 & 300 & 300 \\
\hline$\rho(\mathrm{N} . \mathrm{s} 2 / \mathrm{m} 4)$ & 867 & 867 & 867 & 867 \\
\hline$\mu(\mathrm{N} . \mathrm{s} / \mathrm{m} 2)$ & 0.068 & 0.068 & 0.068 & 0.068 \\
\hline $\mathrm{Cv}(\mathrm{J} / \mathrm{Kg} . \mathrm{c})$ & 1880 & 1880 & 1880 & 1880 \\
\hline$\theta \mathrm{I}($ degree $)$ & 4.85 & 4.96 & 4.86 & 4.96 \\
\hline$\theta r \approx$ & 0 & 0 & 23.37 & 23.83 \\
\hline$\theta \mathrm{e} \approx$ & 88.419 & 76.11 & 88.419 & 76.1 \\
\hline$\beta$ (dim-less) & $2 / 3$ & $2 / 3$ & $2 / 3$ & $2 / 3$ \\
\hline $\operatorname{Ps}(\mathrm{MN} / \mathrm{m} 2)$ & 7.7311 & 12.455 & 7.6978 & 10.027 \\
\hline $\mathrm{e}(\mathrm{mm})$ & 1.4 & 0.3429 & 1.4 & 0.3566 \\
\hline $\mathrm{He}(\mu \mathrm{m})$ & 38.07 & 82.43 & 38.07 & 85.653 \\
\hline $\mathrm{Ke}($ dim-less $)$ & $1 / 36.23$ & $1 / 146$ & $1 / 36.23$ & $1 / 140.2$ \\
\hline
\end{tabular}

\begin{tabular}{lllll}
\hline Bearing & \multicolumn{3}{l}{ Without recess } & With recess \\
\cline { 2 - 5 } Parameters & H. S & P.H. S & H. S & P. H. S \\
\hline $\mathrm{S} \approx$ & 0.1121 & 0.0696 & 0.1126 & 0.0864 \\
$\mathrm{Wl} \approx$ & 0.9881 & 0.6134 & 0.9924 & 0.7619 \\
$\mathrm{Jf}=(1 / \mathrm{Wl}) \approx$ & 1.012 & 1.6303 & 1.0076 & 1.3147 \\
Wo=Wl & 0.9881 & 0.6134 & 0.9924 & 0.7619 \\
wo(KN) & 40 & 40 & 40 & 40 \\
Ws $($ dim-less $)$ & 1.096 & 0.6313 & 1.1017 & 0.8082 \\
ws(KN) & 44.364 & 41.177 & 44.402 & 42.434 \\
$\mathrm{Q}($ dim-less $)$ & 0.003 & 0.1366 & 0.003 & 0.1731 \\
$\mathrm{q}(\mathrm{m} / \mathrm{hr})$ & 1.1275 & 1.2811 & 1.1277 & 1.457 \\
$\mathrm{M}($ dim-less $)$ & 1.6115 & 0.537 & 1.6096 & 0.5344 \\
$\mathrm{~m}(\mathrm{~N} . \mathrm{M})$ & 5.8778 & 7.867 & 5.8709 & 7.5426 \\
$\mathrm{mc}($ dim-less $)$ & 0.2167 & 0.1925 & 0.217 & 0.2163 \\
$\mathrm{dc}(\mathrm{mm})$ & 1.9 & 1.7 & 1.9 & 1.9 \\
$\mathrm{lc} \approx$ & 38 & 34 & 38 & 38 \\
$\mathrm{mo}($ dim-less $)$ & 0.3369 & 0.3183 & 0.3373 & 0.3587 \\
$\mathrm{do}(\mathrm{mm})$ & 2.9 & 2.8 & 2.9 & 3.1 \\
$\mathrm{Pf}(\mathrm{N} . \mathrm{m} / \mathrm{s})$ & 11079 & 14829 & 11066 & 14218 \\
$\mathrm{Pp} \approx$ & 2959 & 5031 & 2950 & 4720 \\
$\mathrm{Pt} \approx$ & 14038 & 19860 & 14016 & 18938 \\
$\lambda \mathrm{c}(\mathrm{N} / \mu \mathrm{m})$ & 203 & 300 & 203 & 296 \\
$\lambda \mathrm{N}(\mathrm{N} / \mu \mathrm{m})$ & 404 & 595 & 404 & 589 \\
$\Delta \mathrm{Tn}($ deg. c) & 20.36 & 28 & 20.26 & 22.9 \\
$\Delta \mathrm{Tth}($ deg. c) & 21.7 & 25.6 & 21.67 & 21.5 \\
\hline & & & & \\
\hline
\end{tabular}

\section{Appendix}

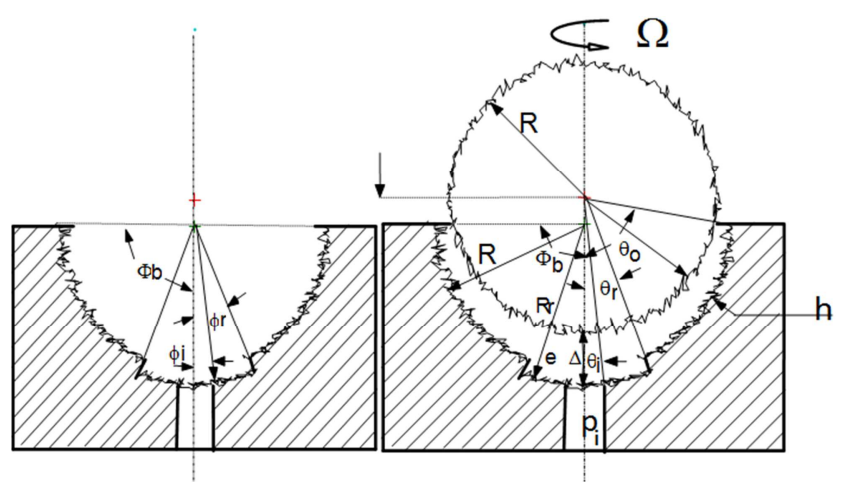

Figure 5. Bearing Configuration.

\section{A1. BEARING GEOMETRY}

A1.1The film thickness and the eccentricity relation

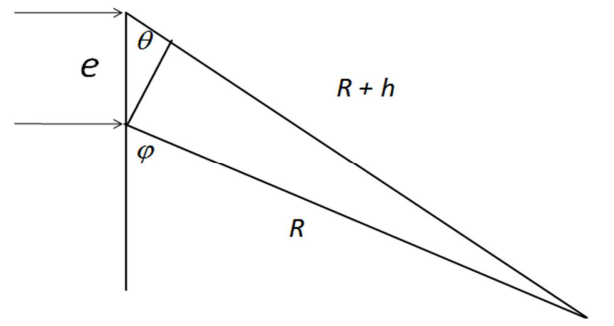

$$
[(R+h)-(e \cos \theta)]^{2}=R^{2}-(e \sin \theta)^{2}
$$

Simply, neglect the term containing $(\sin \theta)^{2}$

Then:

$$
h=e \cos \theta
$$

A1.2 The relation between $(\phi \& \theta \& r)$ 


$$
\phi_{e}=\frac{\eta \pi}{2} \& \phi_{i}=\sin ^{-1}\left(\frac{r}{R}\right)
$$

Generally:

From trigonometry:

$$
\begin{aligned}
& \frac{e}{\sin (\phi-\theta)}=\frac{R}{\sin (\theta)} \\
& \frac{e}{R}=\frac{\sin (\phi-\theta)}{\sin (\theta)}=k_{e}
\end{aligned}
$$

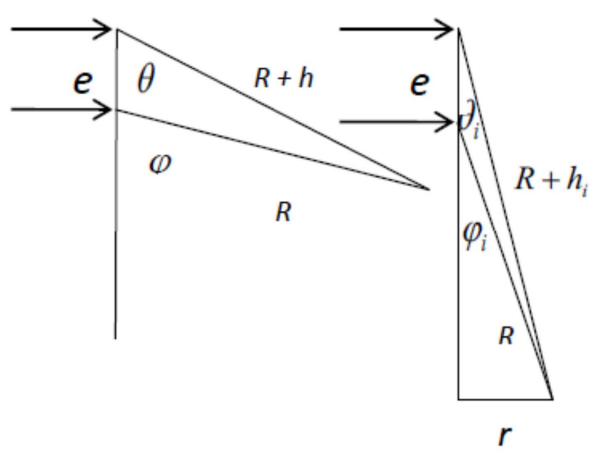

$k_{e}=\frac{\sin \phi \cos \theta-\cos \phi \sin \theta}{\sin (\theta)}=\sin \phi \cot \theta-\cos \phi$

$\cot \theta=\frac{k_{e}+\cos \phi}{\sin \phi}$

$\theta=\cot ^{-1}\left(\frac{k_{e}+\cos \phi}{\sin \phi}\right)$ for the bearing seat

For hemispherical seat:

$$
\begin{aligned}
& \theta_{e}=\cot ^{-1}\left(\frac{k_{e}+\cos \phi_{e}}{\sin \phi_{e}}\right) \\
& \theta_{e}=\cot ^{-1}\left(k_{e}\right)
\end{aligned}
$$

Where:

$$
\begin{gathered}
\phi_{e}=\frac{\eta \pi}{2} \quad \& \eta=1 \\
\frac{d p}{d \theta}=\frac{C\left(1-k_{v} \sin \theta\right)}{\left(h_{o}^{3}+3 \sigma_{o}^{2} h_{o}\right) \sin \theta}+\frac{3}{20} \rho \Omega^{2} R^{2} \sin 2 \theta \\
\text { A2.3 Pressure distribution }(P) \\
P=\frac{\alpha A}{b^{2}+1}\left\{\frac{1}{2 b^{2}} \ln \left(1+b^{2} \sec ^{2} \theta\right)+\ln (\tan \theta)\right\}-\frac{\alpha A k_{v}}{2 b^{2}}\left\{\ln \left(\frac{1+\sin \theta}{1-\sin \theta}\right)+\frac{1}{\sqrt{b^{2}+1}} \ln \left(\frac{\sqrt{b^{2}+1}-\sin \theta}{\sqrt{b^{2}+1}+\sin \theta}\right)\right\}-2 S \cos ^{2} \theta+D
\end{gathered}
$$

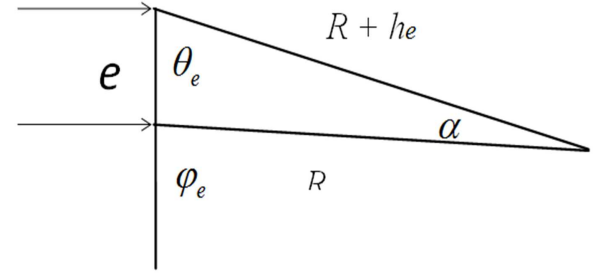

Hemispherical

For partial hemispherical seat:

$$
\theta_{e}=\cot ^{-1}\left(\frac{k_{e}+\cos \phi_{e}}{\sin \phi_{e}}\right)
$$

Where:

$$
\phi_{e}=\frac{\eta \pi}{2} \quad \& \eta \angle 1
$$

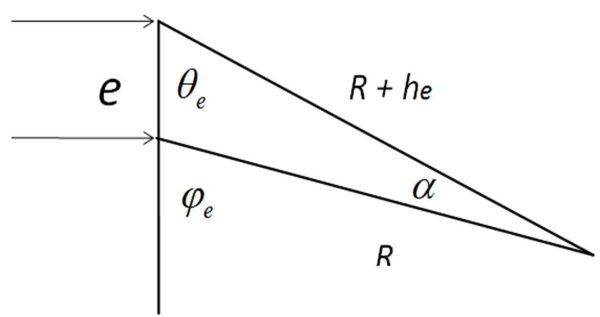

Partial hemispherical

\section{A2. MATHEMATICAL EXPERSSIONS}

A2.1 Simplified Governing Equation

The simple governing equation of this type of bearings considering the centripetal inertia by Dowson [1] is:

$$
h^{3}\left(\frac{d p}{d \theta}-\frac{3}{20} \rho \Omega^{2} R^{2} \sin 2 \theta\right)=\frac{C}{\sin \theta} \text { which was firstly }
$$

modified by Yacout $[9,11]$ to express the surface roughness and finally to express the viscosity variation respectively.

A2.2 Modified Governing Equation

The modified governing equation for this type of bearings and its solution from Yacout [9-11] is:

Where:

$D=B$ at the recess zone.

$D=B \& \alpha=1$ at the seat zone.

$$
B=2 S \cos ^{2} \theta_{e}-A\left[\frac{1}{b^{2}+1}\left\{\frac{1}{2 b^{2}} \ln \left(1+b^{2} \sec ^{2} \theta_{e}\right)+\ln \left(\tan \left(\theta_{e}\right)\right\}-\frac{k_{v}}{2 b^{2}}\left\{\ln \left(\frac{1+\sin \theta_{e}}{1-\sin \theta_{e}}\right)+\frac{1}{\sqrt{b^{2}+1}} \ln \left(\frac{\sqrt{b^{2}+1}-\sin \theta_{e}}{\sqrt{b^{2}+1}+\sin \theta_{e}}\right)\right\}\right]\right.
$$




$$
A=\frac{1+2 S\left(\cos ^{2} \theta_{i}-\cos ^{2} \theta_{e}\right)}{L_{1}-L_{2}}
$$

$L_{1}=\left[\frac{1}{b^{2}+1}\left\{\frac{1}{2 b^{2}} \ln \frac{\left(1+b^{2} \sec ^{2} \theta_{r}\right)}{\left(1+b^{2} \sec ^{2} \theta_{e}\right)}+\ln \frac{\left(\tan \left(\theta_{r}\right)\right.}{\left(\tan \left(\theta_{e}\right)\right.}\right\}-\frac{k_{v}}{2 b^{2}}\left\{\ln \left(\frac{1+\sin \theta_{r}}{1-\sin \theta_{r}} * \frac{1-\sin \theta_{e}}{1+\sin \theta_{e}}\right)+\frac{1}{\sqrt{b^{2}+1}} \ln \left(\frac{\sqrt{b^{2}+1}-\sin \theta_{r}}{\sqrt{b^{2}+1}+\sin \theta_{r}} * \frac{\sqrt{b^{2}+1}+\sin \theta_{e}}{\sqrt{b^{2}+1}-\sin \theta_{e}}\right)\right\}\right]$

$L_{2}=\left[\frac{\alpha}{b^{2}+1}\left\{\frac{1}{2 b^{2}} \ln \frac{\left(1+b^{2} \sec ^{2} \theta_{r}\right)}{\left(1+b^{2} \sec ^{2} \theta_{i}\right)}+\ln \frac{\left(\tan \left(\theta_{r}\right)\right.}{\left(\tan \left(\theta_{i}\right)\right.}\right\}-\frac{\alpha k_{v}}{2 b^{2}}\left\{\ln \left(\frac{1+\sin \theta_{r}}{1-\sin \theta_{r}} * \frac{1-\sin \theta_{i}}{1+\sin \theta_{i}}\right)+\frac{1}{\sqrt{b^{2}+1}} \ln \left(\frac{\sqrt{b^{2}+1}-\sin \theta_{r}}{\sqrt{b^{2}+1}+\sin \theta_{r}} * \frac{\sqrt{b^{2}+1}+\sin \theta_{i}}{\sqrt{b^{2}+1}-\sin \theta_{i}}\right)\right\}\right]$

A2.3.1 Dimensional flow rate $(Q)$

$$
Q=-A=\frac{6 \mu q}{\pi e^{3} \beta p_{s}}
$$

A2.3.2 Flow rate $(Q)$

$$
q=\frac{\pi e^{3} \beta p_{s} Q}{6 \mu}
$$

A2.4 Load carrying capacity (W)

$$
W=\sin ^{2} \theta+2\left[\left(a_{1}+b_{1}\right)-\left(b_{2}-a_{2}\right)\right]
$$

Where:

$$
\begin{aligned}
& a_{1}=A\left[\frac{b^{2}+\cos ^{2} \theta}{4 b^{2}\left(b^{2}+1\right)} \ln \left(b^{2}+\cos ^{2} \theta\right)-\frac{\sin ^{2} \theta}{2\left(b^{2}+1\right)} \ln (\sin \theta)-\frac{\cos ^{2} \theta}{2 b^{2}} \ln (\cos \theta)\right]_{\theta_{e}}^{\theta_{r}}+\frac{S}{2}\left[\cos ^{4} \theta\right]_{\theta_{r}}^{\theta_{e}}-\frac{B}{2}\left[\cos ^{2} \theta\right]_{\theta_{r}}^{\theta_{e}} \\
& a_{2}=\frac{K_{v} A}{4 b^{2}}\left[\frac{\left(\cos ^{2} \theta\right)}{2} \ln \frac{(1-\sin \theta)}{(1+\sin \theta)}-\left(\frac{b^{2}+\cos ^{2} \theta}{2 \sqrt{b^{2}}+1}\right) \ln \frac{\left(\sqrt{b^{2}+1}-\sin \theta\right)}{\left(\sqrt{b^{2}+1}+\sin \theta\right)}\right]_{\theta_{r}}^{\theta_{e}} \\
& b_{1}=A_{r}\left[\frac{b^{2}+\cos ^{2} \theta}{4 b^{2}\left(b^{2}+1\right)} \ln \left(b^{2}+\cos ^{2} \theta\right)-\frac{\sin ^{2} \theta}{2\left(b^{2}+1\right)} \ln (\sin \theta)-\frac{\cos ^{2} \theta}{2 b^{2}} \ln (\cos \theta)\right]_{\theta_{r}}^{\theta_{i}}+\frac{S}{2}\left[\cos ^{4} \theta\right]_{\theta_{i}}^{\theta_{r}}-\frac{B_{r}}{2}\left[\cos ^{2} \theta\right]_{\theta_{i}}^{\theta_{r}} \\
& b_{2}=\frac{K_{v} A_{r}}{4 b^{2}}\left[\frac{\left(\cos ^{2} \theta\right)}{2} \ln \frac{(1-\sin \theta)}{(1+\sin \theta)}-\left(\frac{b^{2}+\cos ^{2} \theta}{2 \sqrt{b^{2}}+1}\right) \ln \frac{\left(\sqrt{b^{2}+1}-\sin \theta\right)}{\left(\sqrt{b^{2}+1}+\sin \theta\right)}\right]_{\theta_{i}}^{\theta_{r}} \\
& A_{r}=\alpha A
\end{aligned}
$$

A2.5 Frictional torque (M)

$$
M=z_{b} M_{r}+M_{s}
$$

Where:

$M_{r}=\left[\frac{\cos ^{2} \theta}{2}+\left(\sigma^{2}-1\right) \ln (\cos \theta)+-\frac{\sigma^{2}}{2 \cos ^{2} \theta}\right]_{\theta_{i}}^{\theta_{r}}-K_{v}\left[\left(\sigma^{2}-1\right)\{\sin \theta-\ln (\tan \theta+\sec \theta)\}-\frac{\sigma^{2}}{2}\{\ln (\sec \theta+\tan \theta)-\sec \theta \tan \theta\}-\frac{\sin ^{3} \theta}{3}\right]_{\theta_{i}}^{\theta_{r}}$ $M_{s}=\left[\frac{\cos ^{2} \theta}{2}+\left(\sigma^{2}-1\right) \ln (\cos \theta)+-\frac{\sigma^{2}}{2 \cos ^{2} \theta}\right]_{\theta_{r}}^{\theta_{e}}-K_{v}\left[\left(\sigma^{2}-1\right)\{\sin \theta-\ln (\tan \theta+\sec \theta)\}-\frac{\sigma^{2}}{2}\{\ln (\sec \theta+\tan \theta)-\sec \theta \tan \theta\}-\frac{\sin ^{3} \theta}{3}\right]_{\theta_{r}}^{\theta_{e}}$

$$
b_{2}=\frac{K_{v} A_{r}}{4 b^{2}}\left[\frac{\left(\cos ^{2} \theta\right)}{2} \ln \frac{(1-\sin \theta)}{(1+\sin \theta)}-\left(\frac{b^{2}+\cos ^{2} \theta}{2 \sqrt{b^{2}}+1}\right) \ln \frac{\left(\sqrt{b^{2}+1}-\sin \theta\right)}{\left(\sqrt{b^{2}+1}+\sin \theta\right)}\right]_{\theta_{i}}^{\theta_{r}}
$$

A2.6 Temperature rise and distribution 


$$
\begin{aligned}
& \frac{d T}{d \theta}=\left(\frac{p_{i}}{\rho c_{v}}\right) \frac{\left(\frac{d P}{d \theta}\right)^{2}-4 S\left(\frac{d P}{d \theta}\right) \sin (2 \theta)+\frac{(S \sin 2 \theta)^{2}}{0.21}}{\left(2 \sin 2 \theta-\frac{d P}{d \theta}\right)}+\frac{(\text { Const }) \sin ^{2} \theta \sec ^{4} \theta\left(1-k_{v} \sin \theta\right)^{2}}{\left(2 \sin 2 \theta-\frac{d P}{d \theta}\right)}=X \\
& \frac{\Delta T}{\Delta \theta}=X \\
& T_{n}=T_{n-1}+X \Delta \theta \\
& T_{1}=T_{i} \\
& \text { Const }=\left(\frac{160 S \mu_{i}^{2}}{\rho p_{i} R^{2} K_{e}^{4}}\right) \\
& q=K_{o}\left[(1-\beta) p_{s}\right]^{\frac{1}{2}} \\
& \Delta \beta=-\frac{\mu\left(q_{2}^{2}-q_{1}^{2}\right)}{K_{o}^{2} p_{s}}
\end{aligned}
$$

A4. CENTRAL PRESSURE RATIO $(\beta)$ A4.1 Capillary tube restrictor

$$
p_{p}=q \cdot p_{s}
$$

$$
\operatorname{Re}_{c}=4 \rho q / \pi \mu d_{c}
$$

$$
\begin{array}{ll}
q=K_{c}\left(p_{s}-p_{i}\right) / \mu=K_{c}(1-\beta) p_{s} / \mu & \text { (a) } \\
K_{c}=\pi d_{c}^{4} / 128 l_{c} \quad, \quad n_{c}=l_{c} / d_{c}=20 & \text { (b) } \\
R e_{c}=2000 \quad \text { for laminar flow } & \text { (c) }
\end{array}
$$

$$
P_{p}=\left(\frac{\pi \beta p_{s}^{2} e^{3}}{6 \mu}\right) Q
$$

$$
\begin{gathered}
m_{c}=d_{c} / d_{i o} \\
a_{1}=\beta^{2}-\beta^{3}
\end{gathered}
$$

Where:

$$
a_{1}=\left(72 n_{c} \mu^{6} \operatorname{Re}_{c}^{3} / e^{6} \rho^{3} p_{s}^{3} Q^{2}\right)
$$

$$
\begin{gathered}
P_{f}=\left(\frac{2 \pi \mu R^{4} \Omega^{2}}{e}\right) M \\
P_{t}=P_{f}+P_{p}
\end{gathered}
$$

\section{A3. NUMERICAL STIFFNESS DERIVATION $(\lambda)$}

$$
\begin{aligned}
& w=a \beta p_{s} W \\
& \lambda=-\frac{d w}{d h} \\
& \lambda=a p_{s}\left[\begin{array}{ll}
\bar{\beta}+\bar{W} \beta
\end{array}\right]
\end{aligned}
$$

Numerically:

$$
\lambda=a p_{s}\left[W \frac{\Delta \beta}{\Delta h}+\beta \frac{\Delta W}{\Delta h}\right]
$$

A3.1 Capillary

$$
\begin{gathered}
q=K_{c} p_{s}[1-\beta] / \mu \\
\Delta \beta=-\frac{\mu\left(q_{2}-q_{1}\right)}{K_{c} p_{s}}=\frac{\mu \Delta q}{K_{c} p_{s}} \\
\Delta h=e_{2} \cos \theta_{2}-e_{1} \cos \theta_{1}
\end{gathered}
$$

A3.2 Orifice

$$
\begin{gathered}
q=K_{o}\left[(1-\beta) p_{s}\right]^{\frac{1}{2}} \\
K_{o}=\frac{\pi C_{d} d_{o}^{2}}{\sqrt{8 \rho}} \\
C_{d}=0.6 \rightarrow \operatorname{Re}_{o}>15 \\
d_{o}>0.5 \mathrm{~mm} \\
\quad m_{o}=d_{o} / d_{i o} \\
a_{2}=\beta^{2}-\beta^{3}
\end{gathered}
$$

Where:

$$
a_{2}=\left(9 C_{d}^{2} \mu^{6} \operatorname{Re}_{o}^{4} / 8 e^{6} \rho^{3} p_{s}^{3} Q^{2}\right)
$$

Differentiating equations $(18,22)$ and equating to zero: Then:

$$
\begin{aligned}
& \frac{d a_{1}}{d \beta}=2 \beta-3 \beta^{2}=0 \\
& \frac{d a_{2}}{d \beta}=2 \beta-3 \beta^{2}=0
\end{aligned}
$$




$$
\beta=2 / 3, \quad a_{1}, a_{2}=4 / 27
$$

A4.3 The restrictor dimensions Determination

$$
\begin{aligned}
K_{c} & =\left(3 \mu q / p_{s}\right) \\
K_{o} & =q /\left(p_{s} / 3\right)^{1 / 2}
\end{aligned}
$$

\section{Nomenclature}

$$
\begin{aligned}
& A=-\left(6 \mu q / e^{3} \pi p_{i}\right) \\
& A_{\text {pwa }}=\text { Projected wet area } \\
& a=\text { Bearing projected area }\left(\pi R^{2}\right) \text {. } \\
& C=-\left(6 \mu_{i} q / \pi\right) \text {. } \\
& c_{v}=\text { Lubricant specific heat } 1880(\mathrm{~J} / \mathrm{Kg} . \mathrm{c}) \\
& E(f)=\text { Expected value of. } \\
& e=\text { Eccentricity } \& e_{r}=\Delta+e \\
& f=\text { Dimensionless friction factor. } \\
& F=\text { Friction factor. } \\
& H=\text { Dimensionless film thickness. } \\
& h=\text { Film thickness }(e \cos \theta) \text {. } \\
& h_{f}=\text { Power facto. } \\
& J_{\mathrm{f}}=\text { Geometry factor }(1 / W) \text {. } \\
& K_{\mathrm{v}}=\text { Constant of viscosity variation } \\
& K_{\mathrm{e}}=(e / R) \& K_{\mathrm{r}}=\left(e_{r} / R\right) \text {. } \\
& \mathrm{L}=\text { Load required to be lifted. } \\
& m=\text { Frictional torque. } \\
& M=\text { Dimensionless frictional torque }\left(m e / 2 \pi \mu \Omega R^{4}\right) \\
& m_{c}=\text { Capillary tube diameter ratio. } \\
& m_{o}=\text { Orifice diameter ratio. } \\
& N=\text { Shaft speed (r.p.s). } \\
& P=\text { Dimensionless pressure }\left(p / p_{i}\right) \\
& p=\text { pressure along the fluid film } \\
& p_{i}=\text { Inlet pressure. } \\
& p_{s}=\text { Supply pressure. } \\
& Q=\text { Dimensionless volume flow rate }(Q=-A) \text {. } \\
& q=\text { Volume flow rate. } \\
& R=\text { Bearing radius. } \\
& R_{e}=\text { Reynolds number. } \\
& S=\text { Speed parameter }\left(3 \rho \Omega^{2} R^{2} / 40 p_{i}\right) \text {. } \\
& \mathrm{T}=\text { Temperature } \\
& W=\text { Dimensionless load carrying capacity }\left(w / \pi R^{2} p_{i}\right) \\
& w=\text { Load carrying capacity. } \\
& z_{b}=\left(e / e_{r}\right) \& \alpha=\left(z_{b}^{3}\right) \\
& \beta=\left(p_{i} / p_{s}\right) \text {. } \\
& \theta=\text { Angle co-ordinate. } \\
& \varphi_{e}=\text { Seat outer rim angle. } \\
& \varphi_{r}=\text { Recess angle } \\
& \theta_{i}=\text { Inlet flow angle. }
\end{aligned}
$$

$\theta_{\mathrm{e}}=$ Outlet flow angle.

$\Delta=$ Recess depth

$\Delta T=$ Temperature rise

$\rho=$ Lubricant density.

$\sigma=$ Dimensionless surface roughness parameter.

$\sigma_{o}^{2}=$ Variance of the film thickness.

$\lambda=$ Bearing stiffness.

$\mu=$ Lubricant viscosity.

$\Omega=$ Rotational speed

\section{Suffix}

$\begin{array}{ll}\mathrm{c} & \text { capillary } \\ \mathrm{o} & \text { orifice } \\ \mathrm{e} & \text { exit, eccentricity } \\ \mathrm{i} & \text { inlet } \\ \text { th } & \text { theoretical } \\ \mathrm{n} & \text { numerical }\end{array}$

\section{References}

[1] Dowson D. and Taylor M. Fluid inertia effect in spherical hydrostatic thrust bearings. ASLE Trans. 1967, 10, 316324.

[2] Rowe W. B. and Stout K. J. Design data and a manufacturing technique for spherical hydrostatic bearings in machine tool applications, Int. J. Mach. Tool Des. Res. 1971, Vol. 11, pp. 293-307.

[3] Cheng and W. B. Rowe, 'A selection strategy for the design of externally pressurized journal bearings', Tribology International, vol. 28, no. 7, pp. 465-474, 1995.

[4] Shijiang Yuan and Decheng Zhou, "Design procedure of an advanced spherical hydrostatic bearing used in rotary forging press", The International Journal of Machine tools and manufacture 1997, Vol. 37, No. 5, pp. 649-656.

[5] Toshiharu Kazama," Optimum design of hydrostatic spherical bearing in fluid film lubrication", Transactions of ASME 2000, Vol. 122, pp. 866-869.

[6] Sharma SC, Jain SC and Bharuka DK. "Influence of Recess Shape on the Performance of a Capillary Compensated Circular Thrust Pad Hydrostatic Bearing", Tribology International 2002 Vol. 35, pp. 347-365.

[7] Singh N, Sharma SC, Jain SC, Reddy SS. "Performance of membrane compensated multi-recess hydrostatic/hybrid flexible journal bearing system considering various recess shapes", Tribology International 2004, Vol. pp. 11-24.

[8] J. R. Lin, Ch. F. Chiang, "Effect of surface roughness and rotational inertia on the optimal stiffness of hydrostatic thrust bearings", International journal of applies mechanics and engineering. 2002, Vol.7, No. 4, pp: 1247- 1261.

[9] Ahmad W. Yacout, Ashraf S. Ismaeel, Sadek Z. Kassab, "The combined effects of the centripetal inertia and the surface roughness on the hydrostatic thrust spherical bearing performance", Tribolgy International Journal 2007, Vol.40, No. 3, 522-532. 
[10] Lu Lihua, Su Hao, Liang Yingchun, Zhang Qiang," Research on Static Stiffness of Hydrostatic Bearing using FluidStructure Interaction Analysis", Procedia Engineering 2012, $29,1304-1308$

[11] Ahmad W. Y. Elescandarany, "The Effect of the fluid film variable viscosity on the hydrostatic thrust spherical bearing performance in the presence of centripetal inertia and surface roughness (Part 1 Un-recessed fitted bearing)", The
International Journal of Mechanical Engineering and Applications2018,Vol.6, No. 1, pp. 1-12.

[12] Ahmad W. Y. Elescandarany, "The Effect of the fluid film variable viscosity on the hydrostatic thrust spherical bearing performance in the presence of centripetal inertia and surface roughness (Part2 Recessed fitted bearing)", The International Journal of Mechanical Engineering and Applications2018, Vol.6, No. 3, pp. 73-90. 